\title{
A simple method for exploring adverse drug events in patients with different primary diseases using spontaneous reporting system
}

Yoshihiro Noguchi", Anri Ueno, Manami Otsubo, Hayato Katsuno, Ikuto Sugita, Yuta Kanematsu, Aki Yoshida, Hiroki Esaki, Tomoya Tachi and Hitomi Teramachi*

\begin{abstract}
Background: Patient background (e.g. age, sex, and primary disease) is an important factor to consider when monitoring adverse drug events (ADEs) for the purpose of pharmacovigilance. However, in disproportionality methods, when additional factors are considered, the number of combinations that have to be computed increases, and it becomes very difficult to explore the whole spontaneous reporting system (SRS). Since the signals need to be detected quickly in pharmacovigilance, a simple exploration method is required. Although association rule mining (AR) is commonly used for the analysis of large data, its application to pharmacovigilance is rare and there are almost no studies comparing AR with conventional signal detection methods.

Methods: In this study, in order to establish a simple method to explore ADEs in patients with kidney or liver injury as a background disease, the AR and proportional reporting ratio (PRR) signal detection methods were compared. We used oral medicine SRS data from the Japanese Adverse Drug Event Report database (JADER), and used AR as the proposed search method and PRR as the conventional method for comparison. "Rule count $\geq 3$ ", "min lift value > 1", and "min conviction value $>1$ " were used as the AR detection criteria, and the PRR detection criteria were "Rule count $\geq 3$ ", "PRR $\geq 2$ ", and " $x^{2} \geq 4$ ".

Results: In patients with kidney injury, the AR method had a sensitivity of 99.58\%, specificity of 94.99\%, and Youden's index of 0.946, while in patients with liver injury, the sensitivity, specificity, and Youden's index were 99.57\%, 94.87\%, and 0.944 , respectively. Additionally, the lift value and the strength of the signal were positively correlated.

Conclusions: It was suggested that computation using AR might be simple with the detection power equivalent to that of the conventional signal detection method as PRR. In addition, AR can theoretically be applicable to SRS other than JADER. Therefore, complicated conditions (patient's background etc.) that must take factors other than the ADE into consideration can be easily explored by selecting the AR as the first screening for ADE exploration in pharmacovigilance using SRS.
\end{abstract}

\footnotetext{
* Correspondence: noguchiy@gifu-pu.ac.jp; teramachih@gifu-pu.ac.jp

Laboratory of Clinical Pharmacy, Gifu Pharmaceutical University,

1-25-4,Daigakunishi, Gifu 501-1196, Japan
}

(c) The Author(s). 2018 Open Access This article is distributed under the terms of the Creative Commons Attribution 4.0 International License (http://creativecommons.org/licenses/by/4.0/), which permits unrestricted use, distribution, and reproduction in any medium, provided you give appropriate credit to the original author(s) and the source, provide a link to the Creative Commons license, and indicate if changes were made. The Creative Commons Public Domain Dedication waiver (http://creativecommons.org/publicdomain/zero/1.0/) applies to the data made available in this article, unless otherwise stated. 


\section{Background}

Recently, due to advances in information technology, large data have begun to be utilized in many fields. Among them is the field of medical monitoring, where numerous risk assessments of drugs have been reported using spontaneous reporting system (SRS), which are based on spontaneous reports of drug-adverse event (AE) pair accumulated and published by regulatory authorities [15].

Various risk assessment methods exist for evaluating adverse drug events (ADEs) based on SRS data, including those based on the proportional reporting ratio (PRR) [6], which is used by the Medicines and Healthcare Products Regulatory Agency (MHRA), and the reporting odds ratio (ROR) [7], which is used by the Netherlands Pharmacovigilance Center Lareb. In addition, a Bayesian Confidence Propagation Neural Network (BCPNN) -based method [8] is used by the World Health Organization (WHO), and a Gamma-Poisson Shrinker (GPS) -based method [9] is used by the United States Food and Drug Administration (FDA). These methods are all signal detection methods used in disproportionality analysis, based on the principle of inequality, focusing on differences in the ratio of the number of reported drug-AE pairs.

If there is no causal relationship between the drug of interest and the $\mathrm{AE}$, the reporting ratio should be approximately the same as the average reporting ratio of other medicines overall. If the reporting ratio of the drug of interest is significantly higher than the average reporting ratio, it is indicative of an $\mathrm{ADE}$, suggesting a causal relationship between the drug and $\mathrm{AE}$ [10].

Since the PRR and the ROR are easy to calculate, it is possible to detect an ADE at an early stage, and since these methods are sensitive, there is little risk of missing a true signal.

These methods use data from an SRS database to create "drug-AE pairs $k \times m$ contingency table". In a typical "drug-AE pairs $2 \times 2$ contingency table" the data are classified into target drug, other drugs, target AE, and other AEs, and the table is used to calculate a risk evaluation index as shown in Fig. 1.

When evaluating ADEs, patient background (e.g. age, sex and primary disease) is another important factor that should be taken into consideration. However, in order to consider additional factors, the drug-AE pairs $2 \times 2$ contingency table must be prepared from a database that extracts data for each factor, and signal indices must be calculated. As a result, the number of combinations becomes enormous, and it is very difficult to implement the calculations efficiently. Thus, a simple signal detection method that allows other factors to be easily considered is urgently needed.

In large data analysis, association rule mining (AR) is aimed at "enumerating interesting patterns hidden in the database" [11-13]. Several analysis methods using AR in pharmacovigilance have recently been proposed [14-17], but there are few reports comparing AR with conventional signal detection methods [17].

Therefore, in this study, in order to establish a simple method for exploring ADEs in patients with kidney injury or liver injury as a primary disease, signal detection using AR and PRR methods was compared.

\section{Methods}

We used the SRS dataset from the 1st quarter of 2004 to the 4th quarter of 2015 from the Japanese Adverse Drug Event Report database (JADER). The JADER was downloaded from Pharmaceuticals and Medical Devices Agency and composed of four tables as follows: DEMO table (with information on gender, age, and weight), DRUG table (with information on suspect drug and concomitant drug), REAC table (with information on AE and outcome), and HIST table (containing medical history of primary diseases and secondary diseases) [18]. Duplicate data and data for nonoral medications were removed from the JADER, and the remaining 184,917 cases were analyzed.

Kidney injury and liver injury can affect drug metabolism, so these primary diseases were considered. The AEs registered in the JADER are represented using the preferred terms (PTs) from the Medical Dictionary for Regulatory Activities (MedDRA). We extracted all of the data for each PT included in the standardized MedDRA Queries (SMQ) for kidney injury and liver injury, which are standard search formulae for MedDRA. The methods used to extract the data from the JADER are illustrated in Fig. 2.

In this study, we used "lift" and "conviction" as the detection criteria for searching the association rule " $\mathrm{A} \cap \mathrm{B} \rightarrow \mathrm{C}$ ", where $A$ is the drug, $B$ is the primary disease, and $C$ is the AE. The calculation methods are shown in Fig. 3 and formula (1) and (2).

$$
\begin{aligned}
\operatorname{lift}(\mathrm{A} \cap \mathrm{B} \rightarrow \mathrm{C}) & =\text { confidence }(\mathrm{A} \cap \mathrm{B} \rightarrow \mathrm{C}) / \operatorname{support}(\mathrm{C}) \\
& =\left(\mathrm{n}_{\mathrm{AB} 1} / \mathrm{n}_{\mathrm{AB}+}\right) /\left(\mathrm{n}_{+1} / \mathrm{n}_{++}\right)
\end{aligned}
$$

$$
\begin{aligned}
\text { conviction } & (\mathrm{A} \cap \mathrm{B} \rightarrow \mathrm{C}) \\
& =(1-\text { Support }(\mathrm{C}) /(1-\text { Confidence }(\mathrm{A} \cap \mathrm{B} \rightarrow \mathrm{C})) \\
& =\left(1-\mathrm{n}_{+1} / \mathrm{n}_{++}\right) /\left(1-\mathrm{n}_{\mathrm{AB} 1} / \mathrm{n}_{\mathrm{AB}+}\right)
\end{aligned}
$$

"Lift" is an index that indicates the relative magnitude of the probability of observing $\mathrm{C}$ under the condition of $A \cap B$, compared to the overall probability of observing $\mathrm{C}$. When the lift value is 1 , the two events $\mathrm{A} \cap \mathrm{B}$ and $\mathrm{C}$ are independent of each other. When the lift value is greater than 1 , the two events $\mathrm{A} \cap \mathrm{B}$ and $\mathrm{C}$ are not 


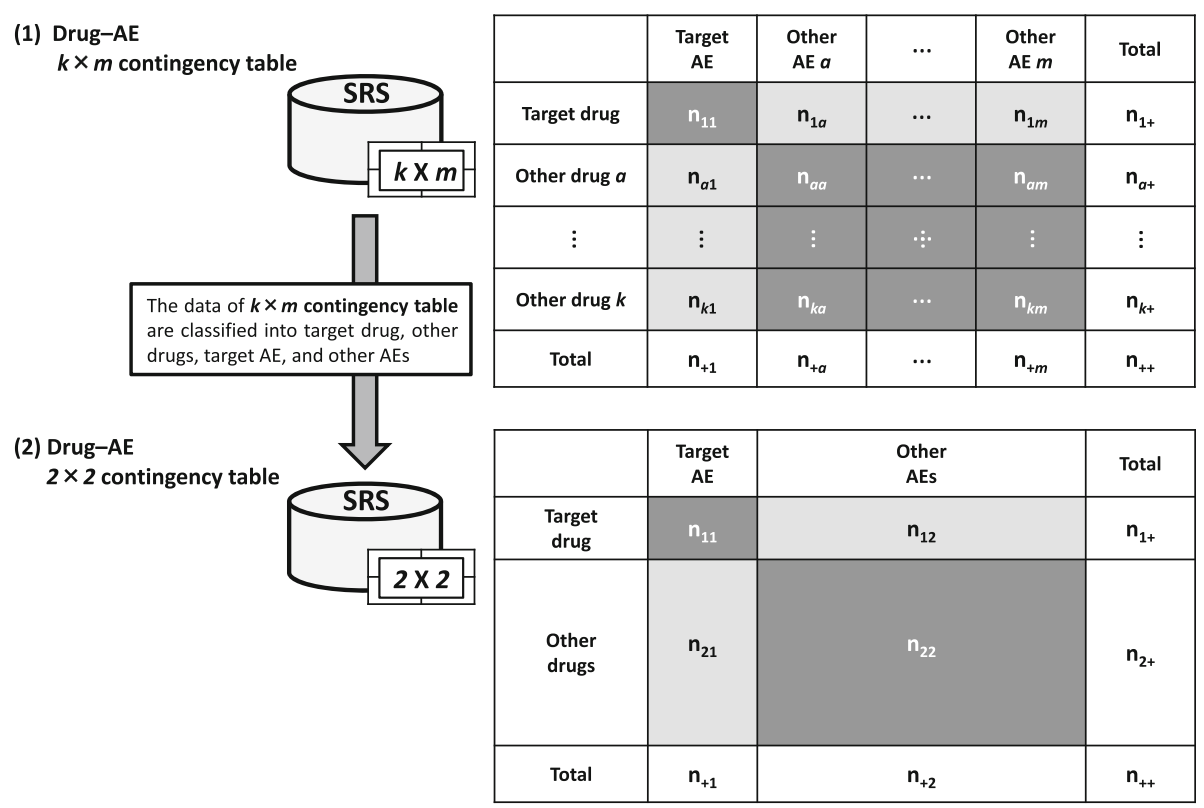

SRS: spontaneous report systems, AE: adverse event, $\mathbf{n}$ : Number of reports,

Fig. 1 Create the drug-AE $k \times m$ contingency table to $2 \times 2$ contingency table for signal detection

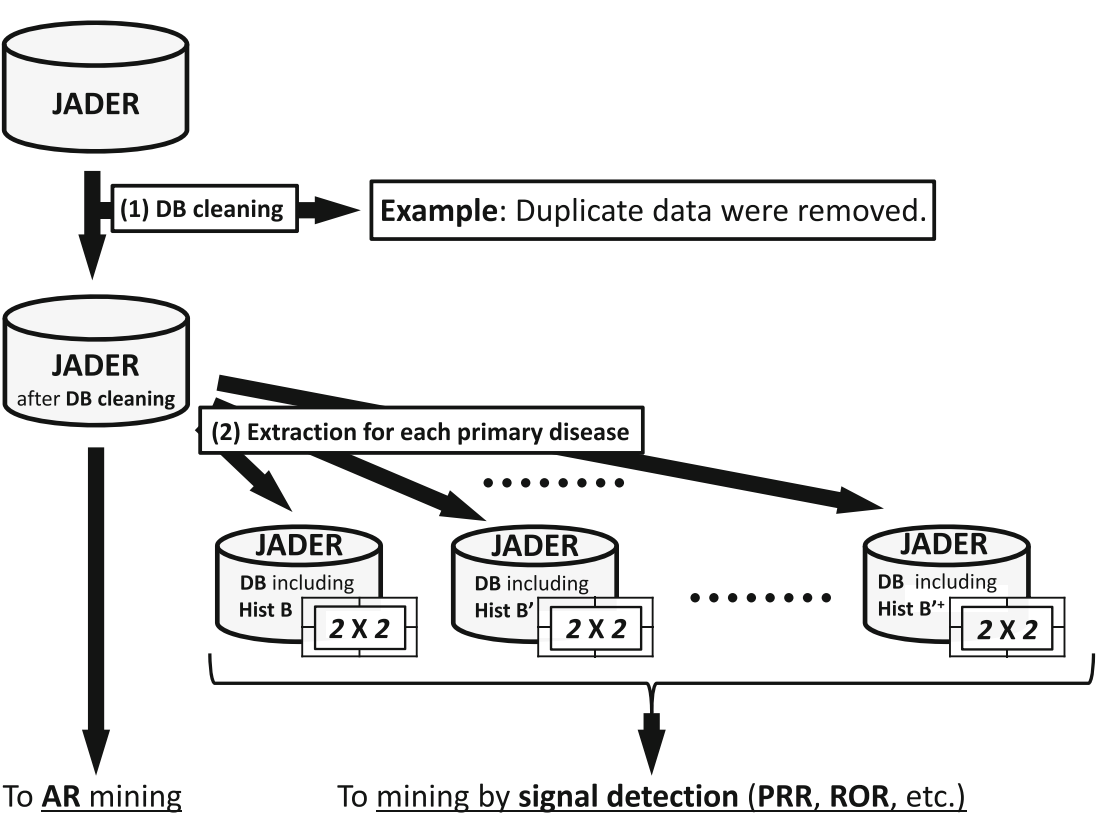

JADER: Japanese Adverse Drug Event Report database, DB: database, Hist: primary disease, $\mathbf{2} \mathbf{X} \mathbf{2}$ : $2 \times 2$ contingency table, AR: association rule mining, PRR: proportional reporting ratio, ROR: reporting odds ratio.

Fig. 2 Database processing for AR mining and mining by signal detection considered about primary disease 


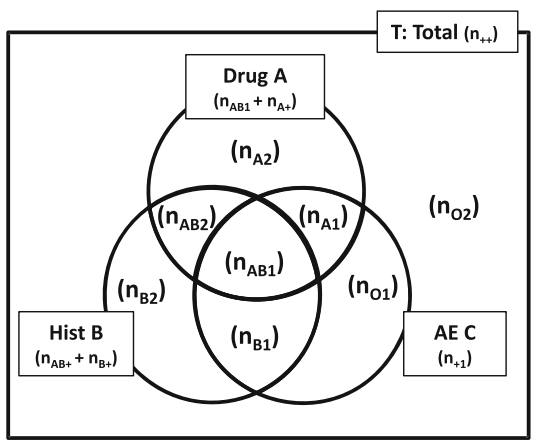

\begin{tabular}{|c|c|c|c|}
\hline & AE C & $\begin{array}{c}\text { All other } \\
\text { AEs }\end{array}$ & Total \\
\hline $\begin{array}{c}\text { Drug A } \\
\text { with Hist B }\end{array}$ & $\mathrm{n}_{\mathrm{AB} 1}$ & $\mathrm{n}_{\mathrm{AB} 2}$ & $\mathrm{n}_{\mathrm{AB}+}$ \\
\hline $\begin{array}{c}\text { Other drugs } \\
\text { with Hist B }\end{array}$ & $\mathrm{n}_{\mathrm{B} 1}$ & $\mathrm{n}_{\mathrm{B} 2}$ & $\mathrm{n}_{\mathrm{B}+}$ \\
\hline $\begin{array}{c}\text { Drug A } \\
\text { without Hist B }\end{array}$ & $\mathrm{n}_{\mathrm{A} 1}$ & $\mathrm{n}_{\mathrm{A} 2}$ & $\mathrm{n}_{\mathrm{A}+}$ \\
\hline $\begin{array}{c}\text { Other drugs } \\
\text { without Hist B }\end{array}$ & $\mathrm{n}_{\mathrm{O} 1}$ & $\mathrm{n}_{\mathrm{O} 2}$ & $\mathrm{n}_{\mathrm{O}+}$ \\
\hline Total & $\mathrm{n}_{+1}$ & $\mathrm{n}_{+2}$ & $\mathrm{n}_{++}$ \\
\hline
\end{tabular}

AR Criteria for this study: Rule counts $\left(n_{A B 1}\right) \geq 3 *$, lift value $>1$ and conviction value $>1$.

*: For PRR $\mathbf{D r u g}_{\text {A with Hist B }}$ signal detection is Rule counts $\left(\mathbf{n}_{\mathrm{AB1}}\right) \geq \mathbf{3}$.

AR: association rule mining, Hist: primary disease, AE: adverse drug event, $\mathbf{n}$ : Number of reports, PRR: proportional reporting ratio.

Fig. 3 The calculation of AR for the Venn diagram and the drug-AE pairs $4 \times 2$ contingency table

independent, and the higher the value, the greater the relevance of the interaction [19].

On the other hand, "conviction" is an indicator that evaluates whether or not the rule makes a wrong prediction, paying particular attention to the exclusion event of the conclusion part of the obtained rule. If the conviction value is large, it is less likely that the conclusion $\mathrm{C}$ is not true for the premise $\mathrm{A} \cap \mathrm{B}$ [20].

In general, lift $>1$ is used as the detection standard for the AR method, but conviction $>1$ was also used in this study. Furthermore, "Rule count $\left(\mathrm{n}_{\mathrm{AB}}{ }_{1}\right) \geq 3$ " was also used according to the detection criteria of the PRR method for comparison.

In addition, in order to verify the accuracy of the signal detected by the AR method, we compared it with the signal detected using the PRR method, which is a conventional signal detection method. As shown in Figs. 2 and 4 and formula (3) and (4), the PRR signal value is calculated from "the drug-AE pairs $2 \times 2$ contingency table" for each case. In the data set, Drug A, Hist B and AE C can be represented by the Venn diagram shown in Fig. 3, but only the part limited to Hist B shown in Fig. 4 is used for calculation of PRR value. The calculation method of PRR is similar to risk ratio which is a general statistical index. According to the criteria of MHRA, the detection standard for the PRR method is "Rule count $\left(\mathrm{n}_{\mathrm{AB}} 1\right) \geq 3$ ", "PRR $\geq$ 2 ", and " $x^{2} \geq 4$ " [6].

Since there were no simulation data in this study, there are no complete data of true risk. Therefore assuming that the signal detected by PRR is true risk, the accuracy of signal detection using the AR method was examined using sensitivity, specificity, Youden's index, positive predictive value (PPV), negative predictive value (NPV), receiver operating characteristic (ROC) curve and area under the ROC

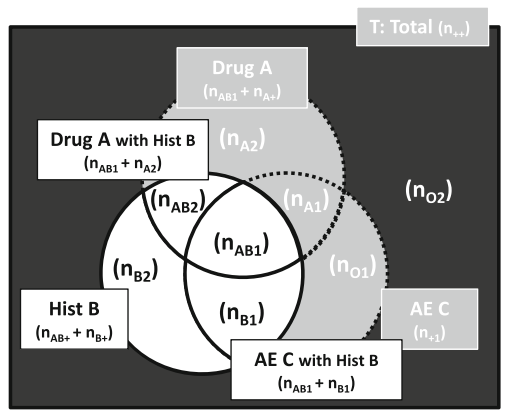

\begin{tabular}{|c|c|c|c|}
\hline & AE C & $\begin{array}{c}\text { All other } \\
\text { AEs }\end{array}$ & $\begin{array}{c}\text { Total } \\
\text { with Hist B }\end{array}$ \\
\hline $\begin{array}{c}\text { Drug A } \\
\text { with Hist B }\end{array}$ & $\mathrm{n}_{\mathrm{AB} 1}$ & $\mathrm{n}_{\mathrm{AB} 2}$ & $\mathrm{n}_{\mathrm{AB}+}$ \\
\hline $\begin{array}{c}\text { Other drugs } \\
\text { with Hist B }\end{array}$ & $\mathrm{n}_{\mathrm{B} 1}$ & $\mathrm{n}_{\mathrm{B} 2}$ & $\mathrm{n}_{\mathrm{B}+}$ \\
\hline $\begin{array}{c}\text { Total } \\
\text { with Hist B }\end{array}$ & $\mathrm{n}_{\mathrm{AB} 1}+\mathrm{n}_{\mathrm{B} 1}$ & $\mathrm{n}_{\mathrm{AB} 2}+\mathrm{n}_{\mathrm{B} 2}$ & $\mathrm{n}_{\mathrm{AB}+}+\mathrm{n}_{\mathrm{B}+}$ \\
\hline
\end{tabular}

Criteria of PRR : Rule counts $\left(n_{A B 1}\right) \geq 3, P R R_{\text {Drug A with Hist } B} \geq 2$ and $\chi^{2} \geq 4$.

PRR: proportional reporting ratio, Hist: primary disease, AE: adverse drug event, $\mathbf{n}$ : Number of reports.

Fig. 4 The calculation of PRR Drug A with Hist B for the Venn diagram and the drug-AE $2 \times 2$ contingency table 
curve (AUC). However, based on the assumption, the cutoff value was not calculated in this study.

Furthermore, the correlation which was investigated using a single regression line between the lift value of the AR method and the signal intensity of the PRR method was also examined (Fig. 4). When examining this correlation, the signal intensity of the PRR is expressed as "log PRR $+\log X^{2 \prime}$, as proposed by Takagi et al. [15].

$$
\begin{aligned}
& \text { PRR }_{\text {Drug A with Hist } \mathrm{B}}=\left(\mathrm{n}_{\mathrm{AB} 1} / \mathrm{n}_{\mathrm{AB}+}\right) /\left(\mathrm{n}_{\mathrm{B} 1} / \mathrm{n}_{\mathrm{B}+}\right) \\
& \mathrm{x}^{2}=\left(\mathrm{n}_{\mathrm{AB}+}+\mathrm{n}_{\mathrm{B}+}\right)\left\{\left|\mathrm{n}_{\mathrm{AB} 1} \mathrm{n}_{\mathrm{B} 2}-\mathrm{n}_{\mathrm{AB} 2} \mathrm{n}_{\mathrm{B} 1}\right|-\left(\mathrm{n}_{\mathrm{AB}+}+\mathrm{n}_{\mathrm{B}+}\right) / 2\right\}^{2} \\
& \quad /\left\{\left(\mathrm{n}_{\mathrm{AB}+} \mathrm{n}_{\mathrm{B}+}\left(\mathrm{n}_{\mathrm{AB} 1}+\mathrm{n}_{\mathrm{B} 1}\right)\left(\mathrm{n}_{\mathrm{AB} 2}+\mathrm{n}_{\mathrm{B} 2}\right)\right\}\right.
\end{aligned}
$$

Data management and analyses were performed using Visual Mining Studio software (version 8.1; Mathematical Systems, Inc. Tokyo, Japan). Drawing the ROC curve and AUC calculation were performed using JMP 11.2.0 (SAS Institute Inc.)

\section{Results}

Among all the cases analyzed (184,917 cases), there were 18,252 cases (24,463 drug-ADE pairs) of kidney injury, and 23,183 cases (23,460 drug-ADE pairs) of liver injury. The number of signals detected for the ADEs using the PRR was 2371 drug-ADE pairs for kidney injury, and 2303 pairs for liver injury.

Table 1 shows the signal detection power of AR for each primary diseases. For kidney injury, the sensitivity was $99.58 \%$, specificity was $94.99 \%$, Youden's index was 0.946, PPV was $68.08 \%$, and NPV was $99.95 \%$. For liver injury, the sensitivity was $99.57 \%$, specificity was $94.87 \%$, Youden's index was 0.944, PPV was $67.88 \%$, and NPV was $99.95 \%$.

Figure 5 shows the ROC curve. For kidney injury, the AUC was 0.974, and for liver injury, the AUC was 0.940. Figure 6 shows the correlation between the lift value and PRR signal intensity. The decision coefficient $\left(R^{2}\right)=0$. 649 for kidney injury and $R^{2}=0.708$ for liver injury.

\section{Discussion}

Although there are several reports of previous studies on the exploration of ADEs using SRS, reports that consider

Table 1 Ability of AR to detect signals for primary diseases

\begin{tabular}{llllll}
\hline $\begin{array}{l}\text { Primary } \\
\text { diseases }\end{array}$ & $\begin{array}{l}\text { Sensitivity } \\
(\%)\end{array}$ & $\begin{array}{l}\text { Specificity } \\
(\%)\end{array}$ & $\begin{array}{l}\text { Youden's } \\
\text { index }\end{array}$ & $\begin{array}{l}\text { PPV } \\
(\%)\end{array}$ & $\begin{array}{l}\text { NPV } \\
(\%)\end{array}$ \\
\hline Kidney injury & 99.58 & 94.99 & 0.946 & 68.08 & 99.95 \\
Liver injury & 99.57 & 94.87 & 0.944 & 67.88 & 99.95
\end{tabular}

$A R$ association rule mining, $P P V$ positive predictive value, NPV negative predictive value factors other than the drug-AE pairs, such as the primary disease affecting the patient, are limited. The reason for this is that in disproportionality methods, considering additional factors requires an enormous number of combinations to be taken into account when signal detection is performed and the respective risk indicators are calculated. Therefore, considering additional factors seems to be impractical as an exploration method.

A potential solution to this issue would be to utilize AR. However, although AR is often used to efficiently analyze large data, there are only a few examples of it being used in the medical field, especially in SRS analysis [14-17]. Signal detection using AR has already been validated as an effective method for the initial identification of "multi-item ADEs" in a study by Harpaz et al. [14]. Furthermore, although information on primary diseases was not included similar to this study, the use of AR in drug-AE pairs was compared with the conventional signal detection method by Wang et al. [17]. Currently, signal detection using AR is not used in pharmacovigilance at regulatory authorities, but is considered very useful for performing complicated analysis considering the patient's background as primary disease.

Therefore, in this study, kidney injury or liver injury as primary disease was considered in addition to the drug$\mathrm{AE}$ pairs, and signal detection was performed using AR method. The conventional PRR method was also used, and the signal detection powers of each method were compared.

In this study, the detection criteria for AR were $\mathrm{n}_{\mathrm{AB} 1} \geq$ 3 , lift $>1$, and conviction $>1$. For kidney or liver injury, in the AR method, both sensitivities were greater than 99\%, and both specificities were greater than $94 \%$. The same detection results were also obtained using the PRR method, indicating that the detection powers of the AR and PRR methods are similar. In addition, the AUC was 0 . 974 for kidney injury and 0.940 for liver injury. These high values suggest that the AR method is also highly accurate.

However, the NPV was greater than $99.9 \%$ for both kidney and liver injury, but the PPV was only $68.08 \%$ for kidney injury and $67.88 \%$ for liver injury.

These results suggest that the AR method may have detected signals that could not be detected by the conventional PRR method. However, unfortunately, we cannot prove our hypothesis, because we did not have "the true risk". The true risk dataset containing "unknown AEs" does not exist.

Because SRS is the result of voluntary reporting and is influenced by reporting bias including underreporting, and the value of the signal easily changes depending on the timing of the analysis, signal detection is not necessarily the true risk, but is limited to the hypothesis of risk. In other words, PRR signals and AR signals are limited to the hypothesis of risk, but they are not the true risk. 


\section{(1) kidney injury}

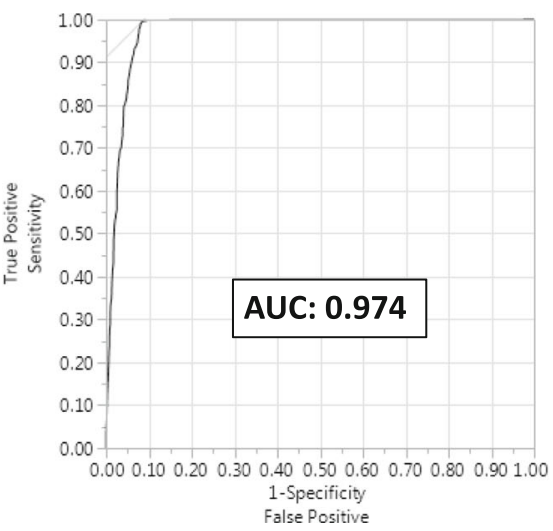

(2) liver injury

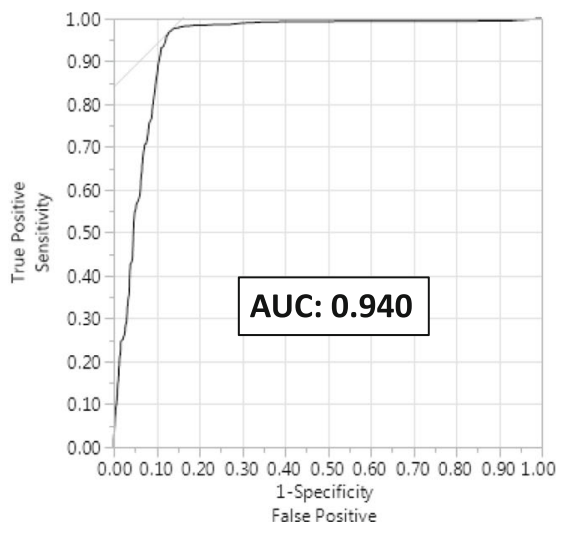

AUC: area under the ROC curve.

Fig. 5 The ROC curve and AUCs for each primary diseases

The detected signals are hypotheses to be clinically noticed until pharmacologic verification is completed. For the AR method to be an alternative to the PRR method, a correlation with the magnitude of the signal value is desirable.

The magnitude of the lift values as AR signals and the PRR signals intensity are positively correlated. Thus, the correlation of the signal values of each method also makes the AR method easy to use for pharmacovigilance.

The conventional PRR method involves extracting data for each of the primary diseases, as shown in Figs. 1, 2 and 4 , and constructing a $2 \times 2$ drug-AE $k \times m$ table. If a similar calculation method as shown in Figs. 2 and 3 that simply creates combinations from the database was used for AR method, the number of the combinations considered would be enormous and it would be difficult to calculate within a realistic time, even if AR method is used.

However, in the AR method, the "apriori algorithm" can be used to reduce the number of calculations. The apriori algorithm is based on the principle that "support of a certain item set is always less than or equal to support of its partial item set" [12]. Therefore, it is unnecessary to calculate the risk index for all combinations, which is required in the conventional method.

In this study, the AR method proposed also requires verification for primary diseases other than kidney injury and liver injury. However, it was suggested that computation using the "apriori algorithm" of AR method might be simple with the detection power equivalent to that of the conventional PRR method.

\section{Conclusion}

The use of post-marketing drugs is complicated, and unlike clinical trials, background factors of patients are diverse. In addition, the frequency of occurrence of ADEs in clinical trial is not known, and there are ADEs that occur over a period longer than the duration of the clinical trial.

SRS analysis using signal detection enables the exploration of unknown ADEs not found in clinical trials and safety assessments in specific populations. It is possible to evaluate safety reflecting the actual clinical use situation.

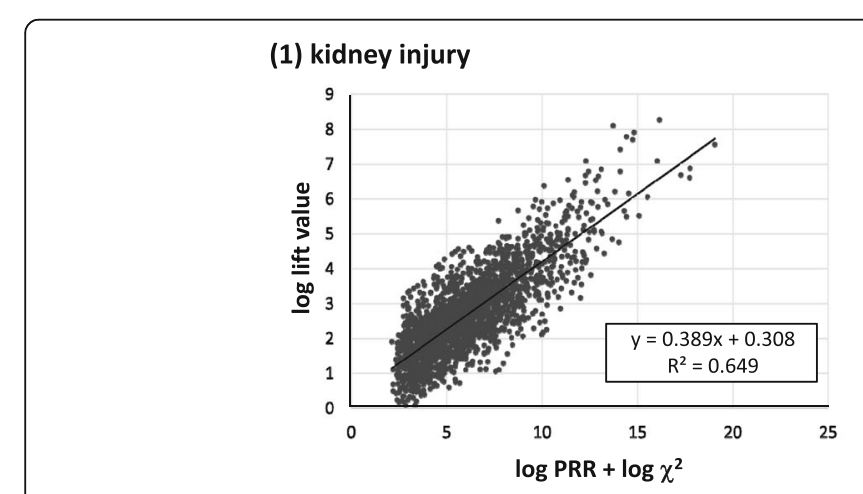

(2) liver injury

Fig. 6 Relation between log lift value and $\log P R R+\log x^{2}$ for each primary diseases

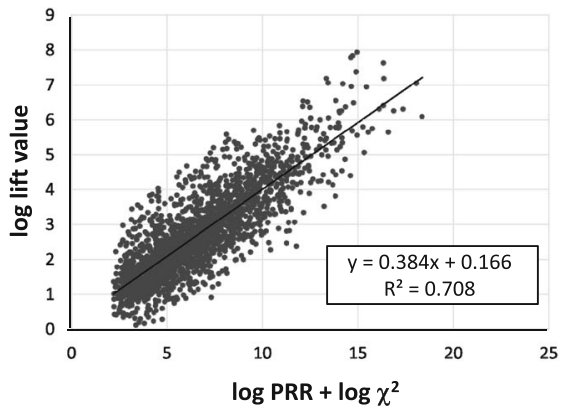


In addition, SRS has played a major role in pharmaepidemiological studies centered on drug safety assessment.

The PRR, which is a conventional signal detection method, is suggestive of ADEs; a similar detection tendency was observed for the AR method. Then, the signal value should be calculated quickly for pharmacological and clinical research. Therefore, in order to reveal the true risk, further pharmacological and clinical research is needed based on the hypothesis obtained. If the method of signal detection is simplified, it will be possible to detect more unknown ADE at an early stage. This is considered important for conducting pharmacological and clinical verification.

In this study, it was suggested that computation using AR method might be simple with the detection power equivalent to that of the conventional signal detection method. In addition, AR method can theoretically be applicable to SRS other than JADER. Therefore, complicated conditions (patient's background etc.) that must take factors other than the AE-drug pairs into consideration can be easily explored by selecting the AR method as the first screening in pharmacovigilance using SRS.

\section{Abbreviations}

ADE: Adverse drug event; AE: Adverse event; AR: Association rule mining; AUC: Area under the ROC curve; BCPNN: Bayesian Confidence Propagation Neural Network; FDA: Food and Drug Administration; GPS: Gamma-Poisson Shrinker; JADER: the Japanese Adverse Drug Event Report database; MedDRA: Medical Dictionary for Regulatory Activities; MHRA: Medicines and Healthcare Products Regulatory Agency; NPV: Negative predictive value; PPV: positive predictive value; PRR: Proportional reporting ratio; PT: Preferred term; ROC: Receiver operating characteristic; ROR: Reporting odds ratio; SMQ: Standardized MedDRA Queries; SRS: Spontaneous reporting system; WHO: World Health Organization

\section{Acknowledgements}

This study was carried out with the aid of JSPS scientific research fund 16 K19175.

\section{Funding}

JSPS scientific research fund 16 K19175.

\section{Availability of data and materials}

Authors do not own the data because the Japanese authority, PMDA, does not does not permit sharing the Japanese Adverse Drug Event Report database (JADER) directly. Data owned by PMDA can be accessed directly here: http:// www.info.pmda.go.jp/fukusayoudb/CsvDownload.jsp (only in Japanese).

\section{Authors' contributions}

Conceived and designed the experiments: YN, HT. Performed the experiments: YN, AU, MO HK. Analyzed the data: YN, IS, YK, AY, HE. Contributed reagents/ materials/analysis tools: YN, HT, TT. Wrote the paper: YN, HT. All authors read and approved the final manuscript.

Ethics approval and consent to participate

Not applicable

\section{Consent for publication}

Not applicable

Competing interests

The authors declare that they have no competing interests.

\section{Publisher's Note}

Springer Nature remains neutral with regard to jurisdictional claims in published maps and institutional affiliations.

Received: 16 May 2017 Accepted: 26 March 2018

Published online: 05 April 2018

\section{References}

1. Poluzzi E, Raschi E, Koci A, Moretti U, Spina E, Behr ER, Sturkenboom M, De Ponti F. Antipsychotics and torsadogenic risk: signals emerging from the US FDA adverse event reporting system database. Drug Saf. 2013;36(6):467-79.

2. Fujimoto M, Hosomi K, Takada M. Statin-associated lower urinary tract symptoms: data mining of the public version of the FDA adverse event reporting system, FAERS. Int J Clin Pharmacol Ther. 2014;52(4):259-66.

3. Noguchi Y, Esaki H, Asano S, Yokoi T, Usui K, Kato M, Saito K, Tachi T, Teramachi $H$. Analysis of effects of the diuretics on levels of blood potassium and blood sodium with angiotensin receptor blockers and thiazide diuretics combination therapy: data Mining of the Japanese Adverse Drug Event Report Database, JADER. Jpn J Pharm Health Care Sci. 2015:41(7):488-96

4. Ali TB, Schleret TR, Reilly BM, Chen WY, Abagyan R. Adverse effects of cholinesterase inhibitors in dementia, according to the Pharmacovigilance databases of the United-States and Canada. PLoS One. 2015;10(12): e0144337. https://doi.org/10.1371/journal.pone.0144337.

5. Gahr M, Connemann BJ, Schönfeldt-Lecuona C, Zeiss R. Sensitivity of Quantitative Signal Detection in Regards to Pharmacological Neuroenhancement. Int J Mol Sci. 2017;18(1) https://doi.org/10.3390/ijms18010101.

6. Evans SJ, Waller PC, Davis S. Use of proportional reporting ratios (PRRs) for signal generation from spontaneous adverse drug reaction reports. Pharmacoepidemiol Drug Saf. 2001;10(6):483-6.

7. van Puijenbroek EP, Bate A, Leufkens HG, Lindquist M, Orre R, Egberts AC. A comparison of measures of disproportionality for signal detection in spontaneous reporting systems for adverse drug reactions. Pharmacoepidemiol Drug Saf. 2002;11(1):3-10.

8. Bate $A$, Lindquist $M$, Edwards $I R$, Olsson $S$, Orre $R$, Lansner $A$, De Freitas RM. A Bayesian neural network method for adverse drug reaction signal generation. Eur J Clin Pharmacol. 1998;54(4):315-21.

9. Szarfman A, Machado SG, O'Neill RT. Use of screening algorithms and computer systems to efficiently signal higher-than-expected combinations of drugs and events in the US FDA's spontaneous reports database. Drug Saf. 2002;25(6):381-92.

10. Zorych I, Madigan D, Ryan P, Bate A. Disproportionality methods for pharmacovigilance in longitudinal observational databases. Stat Methods Med Res. 2013;22(1):39-56.

11. Agrawal R, Imieliński T, Swami A. Mining association rules between sets of items in large databases. ACM SIGMOD Rec. 1993;22(2):207-16.

12. Agrawal R, Srikant R. Fast algorithms for mining association rules. In Proc20thintconf very large databases. 1994;1215:487-99.

13. Lenca $P$, Meyer $P$, Vaillant $B$, Lallich $S$. On selecting interestingness measures for association rules: user oriented description and multiple criteria decision aid. Eur J Oper Res. 2008;184(2):610-26.

14. Harpaz R, Chase HS, Friedman C. Mining multi-item drug adverse effect associations in spontaneous reporting systems. BMC Bioinformatics. 2010;11 https://doi.org/10.1186/1471-2105-11-S9-S7.

15. Shirakuni Y, Okamoto K, Kawashita N, Yasunaga T, Takagi T. Signal detection of drug complications applying association rule learning for StevensJohnson syndrome. J Com Aid Chem. 2009;10:118-27.

16. Fujiwara M, Kawasaki Y, Yamada H. A Pharmacovigilance approach for postMarketing in Japan Using the Japanese adverse drug event report (JADER) database and association analysis. PLoS One. 2016;11(4):e0154425. https:// doi.org/10.1371/journal.pone.0154425

17. Wang C, Guo XJ, Xu JF, Wu C, Sun YL, Ye XF, Qian W, Ma XQ, Du WM, He J. Exploration of the association rules mining technique for the signal detection of adverse drug events in spontaneous reporting systems. PLoS One. 2012;7(7):e40561. https://doi.org/10.1371/journal.pone.0040561.

18. The Japanese Adverse Drug Event Report database (JADER). [http://www. info.pmda.go.jp/fukusayoudb/CsvDownload.jsp] (in Japanese only).

19. Hahsler M, Grün B, Hornik K. Arules - a computational environment for mining association rules and frequent item sets. J Stat Soft. 2005;14(15):1-25.

20. Brin S, Motwani R, Ullman JD, Tsur S. Dynamic itemset counting and implication rules for market basket data. ACM SIGMOD Rec. 1997:26(2):255-64. 